\title{
BMJ Open Web-based and mHealth interventions for intimate partner violence prevention: a systematic review protocol
}

\author{
Elizabeth J Anderson, ${ }^{1}$ Jean McClelland, ${ }^{2}$ Caitlin Meyer Krause, ${ }^{1}$ \\ Keegan C Krause, ${ }^{1}$ David O Garcia, ${ }^{1}$ Mary P Koss ${ }^{1}$
}

To cite: Anderson EJ, McClelland J, Meyer Krause C, et al. Web-based and mHealth interventions for intimate partner violence prevention: a systematic review protocol. BMJ Open 2019;9:e029880. doi:10.1136/ bmjopen-2019-029880

- Prepublication history and additional material for this paper are available online. To view please visit the journal (http:// dx.doi.org/10.1136/bmjopen2019-029880).

Received 18 February 2019

Revised 05 June 2019

Accepted 12 July 2019

\section{Check for updates}

(c) Author(s) (or their employer(s)) 2019. Re-use permitted under CC BY-NC. No commercial re-use. See rights and permissions. Published by BMJ.

${ }^{1}$ College of Public Health, University of Arizona, Tucson, Arizona, USA

${ }^{2}$ Arizona Health Sciences Center, University of Arizona, Tucson, Arizona, USA

Correspondence to

Elizabeth J Anderson;

andersone@email.arizona.edu

\section{ABSTRACT}

Introduction Victims of intimate partner violence (IPV), or those individuals susceptible to IPV victimisation or perpetration, may benefit from participation in primary, secondary or tertiary interventions to address or mitigate exposure to violence despite mixed evidence of IPV intervention effectiveness. However, participation in such programmes is limited by poor access, sociocultural barriers and programme cost. As the world fast approaches universal access to the internet, web-based technologies and low-cost smartphones, new avenues to provide preventive health services including mobile health (mHealth) tools, platforms and services have emerged. The objective of this systematic review is to assess current web-based and mHealth interventions, which include web-based or mobile-based delivery methods for IPV prevention. Interpersonal violence is defined as perpetration or victimisation of a physical, psychological or sexual nature among adults. Interventions may be at the primary, secondary or tertiary level of the public health model.

Methods and analysis This systematic review will incorporate studies focused on any empirical prevention intervention intended for IPV victims or perpetrators of any gender where one or more components is web based or mobile based. Articles will be retrieved from the following academic databases: MEDLINE/PubMed, Embase, CINAHL, Psyclnfo and Open Grey, as well Google Scholar. Results will be limited to articles reporting primary data, published since 1998, and in English, Spanish, Portuguese or French. Data extraction procedures will follow Preferred Reporting Items for Systematic Reviews and Meta-Analyses reporting guidelines. The Mixed Methods Appraisal Tool, a critical appraisal tool, will be used to record ratings of quality and risk of bias among studies selected for inclusion. Content analysis and between-study comparisons will be used to answer the objectives of this review.

Ethics and dissemination Results from this review will be published in an open access format for the benefit of both academic and non-academic audiences, including community organisations and individuals seeking mHealth strategies to reduce and prevent IPV.

Trial registration number CRD42019123006.

\section{INTRODUCTION}

Intimate partner violence (IPV) is defined as any violent or aggressive behaviour that

\section{Strengths and limitations of this study}

This article will systematically report on existing mobile health interventions to reduce intimate partner violence across the globe, including those where intimate partner violence prevention was not the primary intervention goal.

- The findings will identify the platforms that are most successful in populations studied to date.

- The quality of identified primary, secondary and tertiary interventions will be assessed using a validated rating approach applicable to both observational studies and randomised control trials.

- The review will be limited to languages read and understood by investigators, which may result in exclusions of studies published in other widely used languages.

occurs in a close relationship between current or former intimate partners, including sexual, physical or psychological harm that can vary in severity and frequency. ${ }^{1-3}$ IPV can occur with or without sexual intimacy ${ }^{4}$ and may include sexual coercion, sexual touching, refusal to practice safe sex, rape or other non-consensual sex acts with or without physical contact. ${ }^{23}$ IPV can also occur in the form of controlling and isolating behaviours such as limiting the victim's contact with friends and family. ${ }^{25}$ Individuals of any gender may be perpetrators or victims of IPV, though most victims are women and most perpetrators are men. ${ }^{6}$ Men are more likely to report being victimised by low-impact forms of IPV (eg, pushing, shoving and verbal abuse) than women but are much more likely to perpetrate severe forms of IPV such as battery. ${ }^{7}$ Men are much more likely to be studied as perpetrators rather than victims including among sexual minorities. ${ }^{8}$

An estimated $30 \%$ of women around the world have experienced physical and/ or sexual IPV during their lifetime. ${ }^{5} 910$ However, the prevalence of IPV is difficult to estimate due to incompatible data collection 
techniques and tools, non-representative sampling techniques and sociocultural barriers to identifying and disclosing IPV. ${ }^{5}$ Risk of IPV victimisation is elevated in low income and younger populations, those with a history of childhood abuse ${ }^{1112}$ and among immigrants. ${ }^{13}$ Most current reports of IPV are limited to female victims of IPV given that women are more likely to experience severe IPV and are in some instances more likely to have culturally appropriate avenues for reporting IPV victimisation, although IPV is likely widely under-reported across gender. ${ }^{581314}$

IPV is costly to personal and public health in all global contexts. In addition to the direct short-term health consequences of IPV (eg, physical injury), long-term impacts can include post-traumatic stress disorder (PTSD), anxiety disorders and depression ${ }^{3515}$ as well as chronic physiological conditions in the cardiovascular, gastrointestinal, reproductive, musculoskeletal and nervous systems. ${ }^{3}{ }^{12}$ Survivors of IPV may also have increased propensity for health risk behaviours such as smoking, binge drinking, recreational drug use and additional HIV risk factors. ${ }^{15-20}$ At the community level, IPV puts considerable financial strain on medical and social services including care for IPV-related injuries, mental health services, lost workforce productivity and increased demand for criminal justice and child welfare services. ${ }^{3}$ Substantial population and clinic-based evidence shows that overall healthcare consumption is significantly higher among IPV victims, particularly women. ${ }^{121}$

Various primary, secondary and tertiary prevention programmes have been developed to prevent IPV exposure and mitigate health and social consequences after exposure. Primary prevention reduces the incidence of a health threat before it occurs. ${ }^{22}$ Conventional primary prevention programmes addressing IPV often consist of school-based or community-based healthy relationship programmes targeting adolescents and families before victimisation or perpetration occur. ${ }^{12} 2223$ Secondary prevention focuses on early detection after exposure and subsequent treatment in order to triage any resulting negative health consequences or recurrent exposure. Secondary prevention programmes addressing IPV include universal IPV screening in healthcare settings, relocation and/or safe-haven shelters for survivors, access to counselling, medical treatment and legal action to prevent future victimisation. Interventions aimed at reducing perpetration include diversion programmes that promote anger management and de-escalation tactics for perpetrators. Tertiary prevention includes efforts to mitigate the impacts of previous or current experiences of IPV such as counselling for PTSD or recidivism reduction and community reintegration programmes.

Interest in mobile health (mHealth) to deliver public health interventions across mobile devices has increased across disciplines. ${ }^{24}$ mHealth tools are usually but not exclusively web based and often intend to reach audiences that are otherwise reticent to participate in interventions due to the nature of the health issue or barriers to participation. ${ }^{20}$ Barriers to participation include fear of retribution, embarrassment, non-acknowledgement of abuse or violence or perceived cultural taboos about addressing violence. ${ }^{25}$ Protective factors that reduce risk include social support and acceptability of efforts to reduce IPV. ${ }^{26}$ These risk and protective factors may be addressed through web-based efforts that do not rely on conventional in-person interventions.

Existing mHealth and web-based interventions to reduce IPV include novel approaches and methods adapted from evidence-based interventions for online delivery. These approaches include an app-based intervention for college students at risk for dating violence ${ }^{27}$ and educational information to prevent primary, secondary and tertiary victimisation of women adapted from an in-person intervention. ${ }^{28}$ mHealth interventions addressing IPV have been evaluated in observational studies, mixed qualitative and quantitative analyses and randomised control trials (RCTs). ${ }^{29}{ }^{30}$ Ownership of internet-capable devices is highest in high-income countries, where IPV prevention efforts are most likely to be funded or evaluated, though mHealth approaches have been successfully implemented across discipline in low-income and middle-income settings. ${ }^{31}$

\section{Purpose}

To our knowledge, no systematic review has been performed regarding existing mHealth interventions to reduce or mitigate IPV. The purpose of our systematic review is to summarise existing efforts to address IPV using mobile or other web-based programmes and to qualitatively assess their influence at each level of the social ecological model: individual, relationship, community and societal. Our primary objective is to describe how mHealth approaches are being used in IPV prevention using any research approach-quantitative, qualitative or mixed methods-that provides an empirically interpretable estimate of the contextual impact of mHealth. This review will provide insight into: which populations are being served by mHealth interventions to prevent IPV; what, if any, benefits exist for participants; and locate gaps in the literature related to the use of mHealth to address IPV.

\section{METHODS AND ANALYSIS}

This protocol follows the Preferred Reporting Items for Systematic Reviews and Meta-Analyses Protocols (PRISMA-P) 2015 statement $^{32}$; the systematic review will follow both the Preferred Reporting Items for Systematic Reviews and Meta-analyses (PRISMA) statement and the best practices outlined by the Cochrane Collaboration ${ }^{33}$ to ensure transparent reporting.

\section{Inclusion criteria}

Participants

This review will include studies of adults as defined by the study authors (typically 18 years or older) who receive any 
form of intervention related to IPV primary, secondary or tertiary prevention with a web-based, mobile-based or other technology-based delivery component. Interventions intended for either or both victims and perpetrators are included.

\section{Phenomena of interest}

This review will include studies with any sort of intervention regarding primary, secondary or tertiary prevention of IPV victimisation or perpetration and any outcome related to its reduction including barriers and facilitators. The review aims to elucidate characteristics that distinguish web-based IPV prevention programmes; make-up of intended audiences and characteristics of web-based prevention programmes result in highest completion and programme acceptance. The primary outcome focuses on results of participation in programming where IPV prevention is either a direct or indirect goal of the intervention and contains one or more web-based delivery component. Secondary outcomes of interest are acceptability of different aspects of web-based primary, secondary or tertiary IPV prevention programmes, evaluation of causes of dropout and evaluation of studies stratified by racial/ ethnic/gender group. Interventions that focus on other domains of health behaviour (eg, HIV risk reduction) or relationship health (eg, couple therapy) that include IPV prevention as a secondary goal or outcome will also be included.

\section{Context}

This review will consider studies that have one or more elements that take place via a web-based, mobile-based or other technology-based platform (ie, one where the outcome of the intervention depended on the use of a platform such as a computer, cell phone or tablet). No geographic limiters will be considered.

\section{Type of studies}

This review will include any sort of study including RCTs, quasirandomised control trials, pre/post assessments or observational studies that include participant satisfaction data or other outcome data. We will also include relevant cross-sectional surveys if they relate to participation in a qualifying mHealth programme or intervention. Both qualitative and quantitative data will be considered. Comparators will be 'no intervention', any 'pre' data collected before the intervention and/or routine care. Qualitative studies will be included only if they examined and interpreted a measure such as acceptability.

\section{Exclusion criteria}

Both experimental (randomized controlled trial; RCT) and pre-experimental or quasiexperimental studies will be excluded if their focus includes the following: family or interpersonal violence outside of intimate relationship contexts; use a computer-based delivery method that has no bearing on the outcome of interest (eg, web-based recruitment for a face-to-face intervention or computer-assisting survey interviewing); and/or are not available in a language read by study authors.

\section{Search strategy}

Initial searches were performed in MEDLINE/PubMed and Embase databases using keywords 'intimate partner violence', 'intervention' and 'mHealth' were used to harvest keywords, Mesh and Emtree terms, and publication types from resulting titles and abstracts. The search strategy was iteratively refined to ensure that relevant articles were identified. Both published and unpublished studies will be considered. Only studies published in English, Spanish, Portuguese or French will be included given capabilities and limitations of the study team.

The following databases will be included in the search: MEDLINE/PubMed, Embase, PsycInfo, CINAHL and the Cochrane Central Register of Controlled Trials. Articles published between 1998 and 2019 will be screened. The year 1998 was selected as the lower date limit because it is unlikely that any web-based health interventions were performed or assessed before that time. ${ }^{34}$ The search strategy for MEDLINE/PubMed is published in the online supplementary appendix. Adaptations to the MEDLINE/PubMed search strategy will be made for each included database in collaboration with the research librarian (JM). Unpublished studies including theses, dissertations and grey literature will be searched for via the OpenGrey and ProQuest Dissertations and Theses databases. Additionally, the first 100 results from Google Scholar (scholar.google.com) using the specified search terms will be included in review given the high precision and coverage of Google Scholar relative to bibliographic databases. ${ }^{35}$

\section{Study selection}

Following the search, all identified article information will be collated and uploaded into EndNote X8.2 (Clarivate Analytics, Pennsylvania, USA) and deduplicated. Where multiple citations report on the same data, only the most recent or complete citation will be included. Titles and abstracts will be independently screened by two researchers (EJA, CMK or KCK) with the third researcher arbitrating any discordant decisions. Studies marked for potential inclusion will be flagged for fulltext review. Studies without an abstract will be flagged for full-text review. Two researchers will independently screen each of the selected citations for inclusion (EJA, CMK or KCK). Any full-text article that does not meet the inclusion criteria will be recorded along with the reason for exclusion, in accordance with PRISMA guidelines. Any disagreements between the two independent reviewers assigned to any given article will be resolved through discussion and or arbitration with the third reviewer or else other members of the research team (MPK). The results of the search will be reported in full in the final systematic review and presented in a PRISMA flow diagram. ${ }^{32}$ 


\section{Assessment of methodological quality and risk of bias}

All studies, regardless of the results of their methodological quality, will undergo data extraction and synthesis (where possible). Individual studies will be scored following Assessing the Methodological Quality of Systematic Reviews 2 guidelines. ${ }^{36}$ The Mixed Methods Appraisal Tool (MMAT) will be used given the likely inclusion of observational studies, qualitative studies and RCTs. The MMAT contains five distinct, validated subscales to evaluate a wide range of empirical studies (ie, qualitative, quantitative RCTs, quantitative non-randomised trials, quantitative descriptive and mixed methods) where each subscale evaluates the methodological quality of the study in question. ${ }^{37}$

\section{Data extraction}

Included studies will be assessed by two independent reviewers (EJA, CMK or KCK) using a data extraction table created in Excel. The data extracted will include specific details about the populations, context, culture, geographical location, study methods and the phenomena of interest relevant to the review objective, in addition to details of the study design, target population characteristics and sample size, data analysis methods, context (eg, community based vs academic), web-based or mobilebased intervention delivery methods, measure (ie, instrument) of IPV used, type/severity of IPV, primary and secondary outcomes of interest and effect size, where reported. Primary outcomes reported by included experimental studies are likely to include reduction in IPV experiences or rate of entry into care, as well as intervention feasibility in pre-experimental or quasiexperimental studies including pilot studies. Secondary outcomes reported by included trials may include attitudes towards violence or changes in mental wellness scores (eg, depression). Findings, and their illustrations, will be extracted and assigned a level of credibility. Any disagreements that arise between the reviewers will be resolved through discussion or with a third reviewer (MPK). Authors of papers will be contacted to request missing or additional data, where required.

\section{Data synthesis}

Study results and extracted data will be synthesised by categorising findings based on similarities and differences of meaning and using meta-aggregation (crossstudy generalisations). Where possible, assembled findings will be stratified into meaningful categories such as global setting, type of target population and level of scientific evidence provided (eg, RCT vs observational study). Findings will be synthesised to produce one comprehensive summary. Tables will be used where possible, followed by qualitative, narrative descriptions of findings. The summary of findings will include the publication title and year, outcome of interest, study type and context. Comparisons will be made relative to the differences in IPV reporting rates based on population type, measure of IPV prevalence used and variety in severity/ type of IPV experienced by participants (eg, assessment of the impact of intervention types given the severity of IPV). Due to the likely heterogeneity of study design and populations, no quantitative analysis (eg, meta-analysis) is planned. However, where experimental studies use the same primary (eg, IPV experiences) or secondary (eg, depression or anxiety) outcome measures and instruments, these results will be meta-aggregated and stratified by population type, method of intervention delivery and/or severity of IPV. Pre-experimental or quasiexperimental studies or studies using qualitative methods will be meta-aggregated where possible based on comparable factors including method of intervention delivery, dropout rate and intervention characteristics such as participant demographics and length of intervention. Meta-aggregation will be used as the foundation of our data synthesis plan because it estimates the influence of individual studies in terms of their applied significance in the cumulative evidence.

\section{Ethics and dissemination}

The findings of this review may be useful to academic researchers, community-based organisations and lay activists seeking to reduce or mitigate IPV using novel mHealth platforms, tools and methods. Our findings may additionally highlight gaps in knowledge about the effectiveness, efficacy or global applicability of mHealth in IPV prevention.

The systematic review process will follow PRISMA as well as the data extraction processes outlined by the Cochrane Collaboration. ${ }^{32}$ This review protocol follows the PRISMA-P guidelines. ${ }^{33}$ However, given the current evidence about the types and locations of conventional IPV prevention interventions, it is unlikely that we will be able to make meaningful inferences about many global populations, including ethnic, racial, gender or sexual minorities, those from low-income or middle-income countries. The inclusion of research librarian (JM) minimises the possibility of missing any relevant publications, yet language limitations of the study team prevent screening studies published in most languages if no English translation is available.

After completion of this review, we will present our findings at academic meetings and, if relevant, to community-based organisations or partners interested in using mHealth tools to provide IPV prevention services.

In conclusion, this review is the first to assess existing efforts to prevent IPV using one or more mobile elements. The results and discussion will descriptions including integration across studies, abstract conclusions about the state of collected data, advances needed to fill gaps and recommendations for a future research agenda including the most important deficits that need to be addressed. The resultant information will be helpful for the future development or adaptation of IPV prevention services given an increasing global emphasis on web-based public health prevention efforts. 


\section{Patient and public involvement}

Patient and public involvement are not appropriate for this work. The research question answered in this work will explore patient preference for prevention approaches and the acceptability of web-based interventions in a hard-to-reach population. No patients were recruited for this study; there was no public involvement. The results of this study will be publicly disseminated in an open access, peer-reviewed journal that can be accessed by community-based organisations or individuals interested in IPV prevention.

\section{Collaborators A Welter.}

Contributors EJA, DOG and MPK were responsible for the conceptualisation of the research question, approach and rationale. EJA and JMC developed the methods to be used for this review. CMK and KCK provided initial research into existing literature and developed the introduction to this manuscript. EJA prepared the first draft of this manuscript, which was reviewed and revised by MPK and DOG. All authors read and approved the final manuscript.

Funding The authors have not declared a specific grant for this research from any funding agency in the public, commercial or not-for-profit sectors.

Competing interests None declared.

Patient consent for publication Not required.

Provenance and peer review Not commissioned; externally peer reviewed.

Open access This is an open access article distributed in accordance with the Creative Commons Attribution Non Commercial (CC BY-NC 4.0) license, which permits others to distribute, remix, adapt, build upon this work non-commercially, and license their derivative works on different terms, provided the original work is properly cited, appropriate credit is given, any changes made indicated, and the use is non-commercial. See: http://creativecommons.org/licenses/by-nc/4.0/.

\section{REFERENCES}

1. Campbell JC. Health consequences of intimate partner violence. The Lancet 2002;359:1331-6.

2. Krug EG, Mercy JA, Dahlberg LL, et al. The world report on violence and health. The Lancet 2002;360:1083-8.

3. Centers for Disease Control and Prevention (CDC). Preventing intimate partner violence [factsheet] 2017.

4. Centers for Disease Control and Prevention (CDC). Intimate partner violence 2018.

5. Devries KM, Mak JYT, García-Moreno C, et al. Global health. the global prevalence of intimate partner violence against women. Science 2013;340:1527-8.

6. Sugg N. Intimate partner violence: prevalence, health consequences, and intervention. 2015. Medical Clinics, 99(3):629-497.

7. Mills TJ, Avegno JL, Haydel MJ. Male victims of partner violence: prevalence and accuracy of screening tools. J Emerg Med 2006;31:447-52.

8. Carvalho AF, Lewis RJ, Derlega VJ, et al. Internalized sexual minority stressors and same-sex intimate partner violence. J Fam Violence 2011;26:501-9.

9. García-Moreno C, Jansen HA, Ellsberg M. WHO multi-country study on women's health and domestic violence against women: initial results on prevalence, health outcomes and women's responses. World Health Organization, 2005.

10. Yakubovich AR, Stöckl H, Murray J, et al. Risk and protective factors for intimate partner violence against women: systematic review and meta-analyses of Prospective-Longitudinal studies. Am J Public Health 2018;108:e1-11.

11. Capaldi DM, Knoble NB, Shortt JW, et al. A systematic review of risk factors for intimate partner violence. Partner Abuse 2012;3:231-80.

12. Prevention CfDCa. Intimate partner violence: risk and protective factors for perpetration 2018.
13. Burman E, Chantler K. Domestic violence and minoritisation: legal and policy barriers facing minoritized women leaving violent relationships. Int J Law Psychiatry 2005;28:59-74.

14. Sutter ME, Rabinovitch AE, Trujillo MA, et al. Patterns of intimate partner violence victimization and Perpetration among sexual minority women: a latent class analysis. Violence Against Women 2019;25:572-92.

15. Breiding MJ, Black MC, Ryan GW. Chronic disease and health risk behaviors associated with intimate partner violence-18 U.S. states/ territories, 2005. Ann Epidemiol 2008;18:538-44.

16. Coker AL, Davis KE, Arias I, et al. Physical and mental health effects of intimate partner violence for men and women. Am J Prev Med 2002;23:260-8.

17. Lemon SC, Verhoek-OftedahI W, Donnelly EF. Preventive healthcare use, smoking, and alcohol use among Rhode island women experiencing intimate partner violence. J Womens Health Gend Based Med 2002;11:555-62.

18. Wu E, El-Bassel N, Witte SS, et al. Intimate partner violence and HIV risk among urban minority women in primary health care settings. AIDS Behav 2003;7:291-301.

19. Oram S, Khalifeh H, Howard LM. Violence against women and mental health. Lancet Psychiatry 2017;4:159-70.

20. Overstreet NM, Okuyan M, Fisher CB. Perceived risks and benefits in IPV and HIV research: listening to the voices of HIV-positive African American women. J Empir Res Hum Res Ethics 2018;13:511-24.

21. White D, McMillan L. Statutory response to sexual violence. The Routledge Handbook of Gender and Violence, 2018.

22. Coker AL. Primary Prevention of Intimate Partner Violence for Women's Health. J Interpers Violence 2004:19:1324-34.

23. Niolon PH. Preventing intimate partner violence across the lifespan: a technical package of programs, policies, and practices. Government Printing Office, 2017.

24. Krishna S, Boren SA, Balas EA. Healthcare via cell phones: a systematic review. Telemedicine and e-Health 2009;15:231-40.

25. Arango DJ, Morton M, Gennari F, et al. Interventions to prevent or reduce violence against women and girls: a systematic review of reviews 2014.

26. Briones-Vozmediano E, La Parra D, Vives-Cases C. Barriers and facilitators to effective coverage of intimate partner violence services for immigrant women in Spain. Health Expect 2015;18:2994-3006.

27. Glass N, Clough A, Case J, et al. A safety APP to respond to dating violence for College women and their friends: the MyPlan study randomized controlled trial protocol. BMC Public Health 2015; $15: 871$

28. Villegas N, Santisteban D, Cianelli R, et al. The development, feasibility and acceptability of an Internet-based STI-HIV prevention intervention for young Chilean women. Int Nurs Rev 2014;61:55-63.

29. Koziol-McLain J, Vandal AC, Nada-Raja S, et al. A web-based intervention for abused women: the New Zealand isafe randomised controlled trial protocol. BMC Public Health 2015;15:56.

30. Tarzia L, Valpied J, Koziol-McLain J, et al. Methodological and ethica challenges in a web-based randomized controlled trial of a domestic violence intervention. J Med Internet Res 2017;19:e94.

31. Gurman TA, Rubin SE, Roess AA. Effectiveness of mHealth behavior change communication interventions in developing countries: a systematic review of the literature. J Health Commun 2012;17 Suppl 1:82-104.

32. Moher D, Shamseer L, Clarke M, et al. Preferred reporting items for systematic review and meta-analysis protocols (PRISMA-P) 2015 statement. Syst Rev 2015;4:1.

33. van Tulder M, Furlan A, Bombardier C, et al. Updated method guidelines for systematic reviews in the Cochrane collaboration back review group. Spine 2003;28:1290-9.

34. Strecher V. Internet methods for delivering behavioral and healthrelated interventions (eHealth). Annu Rev Clin Psychol 2007;3:53-76.

35. Bramer WM, Giustini D, Kramer BM, et al. The comparative recall of Google Scholar versus PubMed in identical searches for biomedical systematic reviews: a review of searches used in systematic reviews. Syst Rev 2013;2:115.

36. Shea BJ, Reeves BC, Wells G, et al. AMSTAR 2: a critical appraisal tool for systematic reviews that include randomised or nonrandomised studies of healthcare interventions, or both. BMJ 2017;358

37. Hong QN, Pluye P. Mixed methods appraisal tool (MMAT), version 2018. IC Canadian Intellectual Property Office, Industry Canada, 2018. 